\title{
A new reaction pathway in the ester aminolysis catalyzed by glymes and crown ethers
}

Nuno Basilio, Luís García-Río, Juan C. Mejuto, Moisés Pérez-Lorenzo

\begin{tabular}{|l|r|}
\hline Influence of $12 \mathrm{C} 4$ on the $k_{o b s}$ for butylaminolysis of NPA at $25^{\circ} \mathrm{C}$ & $\mathrm{S} 2$ \\
\hline Influence of $15 \mathrm{C} 5$ on the $k_{o b s}$ for butylaminolysis of NPA at $25^{\circ} \mathrm{C}$ & $\mathrm{S} 4$ \\
\hline Influence of $18 \mathrm{C} 6$ on the $k_{o b s}$ for butylaminolysis of NPA at $25^{\circ} \mathrm{C}$ & $\mathrm{S} 6$ \\
\hline Influence of G2 on the $k_{o b s}$ for butylaminolysis of NPA at $25^{\circ} \mathrm{C}$ & $\mathrm{S} 8$ \\
\hline Influence of G3 on the $k_{o b s}$ for butylaminolysis of NPA at $25^{\circ} \mathrm{C}$ & $\mathrm{S} 10$ \\
\hline Influence of G4 on the $k_{o b s}$ for butylaminolysis of NPA at $25^{\circ} \mathrm{C}$ & $\mathrm{S} 12$ \\
\hline Influence of G5 on the $k_{o b s}$ for butylaminolysis of NPA at $25^{\circ} \mathrm{C}$ & $\mathrm{S} 15$ \\
\hline Influence of G4 on the $k_{o b s}$ for butylaminolysis of NPA at $-20^{\circ} \mathrm{C}$ & $\mathrm{S} 18$ \\
\hline Influence of G4 on the $k_{o b s}$ for butylaminolysis of NPA at $45^{\circ} \mathrm{C}$ & $\mathrm{S} 21$ \\
\hline Influence of butylamine on the $k_{o b s}$ for butylaminolysis of $\mathrm{NPA}$ at $25^{\circ} \mathrm{c}$ & $\mathrm{S} 24$ \\
\hline
\end{tabular}


Table S-1. Influence of $n$-butylamine concentration for the aminolysis of NPA in chlorobenzene. $[12 \mathrm{C} 4]=0.10 \mathrm{M} ;[\mathrm{NPA}]=5 \times 10^{-5} \mathrm{M} . \mathrm{T}=25^{\circ} \mathrm{C}$.

\begin{tabular}{cc}
\hline $\left.\mathrm{BuNH}_{2}\right], \mathbf{M}$ & $\boldsymbol{k}_{\text {obs }}, \mathbf{s}^{-1}$ \\
\hline 0.02 & $1.055 \times 10^{-4}$ \\
0.04 & $2.678 \times 10^{-4}$ \\
0.08 & $7.686 \times 10^{-4}$ \\
0.10 & $1.119 \times 10^{-3}$ \\
0.15 & $2.215 \times 10^{-3}$ \\
0.30 & $8.496 \times 10^{-3}$ \\
0.40 & $1.511 \times 10^{-2}$ \\
\hline
\end{tabular}

Table S-2. Influence of $n$-butylamine concentration for the aminolysis of NPA in chlorobenzene. $[12 \mathrm{C} 4]=0.25 \mathrm{M} ;[\mathrm{NPA}]=5 \times 10^{-5} \mathrm{M} . \mathrm{T}=25^{\circ} \mathrm{C}$.

\begin{tabular}{cc}
\hline$\left[\mathrm{BuNH}_{2}\right], \mathbf{M}$ & $\boldsymbol{k}_{\text {obs }}, \mathrm{s}^{-1}$ \\
\hline 0.02 & $2.163 \times 10^{-4}$ \\
0.04 & $5.014 \times 10^{-4}$ \\
0.08 & $1.303 \times 10^{-3}$ \\
0.10 & $1.818 \times 10^{-3}$ \\
0.15 & $3.416 \times 10^{-3}$ \\
0.30 & $1.099 \times 10^{-2}$ \\
0.40 & $1.835 \times 10^{-2}$ \\
\hline
\end{tabular}


Table S-3. Influence of $n$-butylamine concentration for the aminolysis of NPA in chlorobenzene. $[12 \mathrm{C} 4]=0.50 \mathrm{M} ;[\mathrm{NPA}]=5 \times 10^{-5} \mathrm{M} . \mathrm{T}=25^{\circ} \mathrm{C}$.

\begin{tabular}{cc}
\hline $\left.\mathrm{BuNH}_{2}\right], \mathbf{M}$ & $\boldsymbol{k}_{\boldsymbol{o b s}}, \mathbf{s}^{-1}$ \\
\hline 0.02 & $4.299 \times 10^{-4}$ \\
0.04 & $9.581 \times 10^{-4}$ \\
0.08 & $2.311 \times 10^{-3}$ \\
0.10 & $3.070 \times 10^{-3}$ \\
0.15 & $5.187 \times 10^{-3}$ \\
0.30 & $1.592 \times 10^{-2}$ \\
0.40 & $2.700 \times 10^{-2}$ \\
\hline
\end{tabular}

Table S-4. Influence of $n$-butylamine concentration for the aminolysis of NPA in chlorobenzene. $[12 \mathrm{C} 4]=0.72 \mathrm{M} ;[\mathrm{NPA}]=5 \times 10^{-5} \mathrm{M} . \mathrm{T}=25^{\circ} \mathrm{C}$.

\begin{tabular}{cc}
\hline $\left.\mathrm{BuNH}_{2}\right], \mathbf{M}$ & $\boldsymbol{k}_{\text {obs }}, \mathbf{s}^{-1}$ \\
\hline 0.02 & $6.425 \times 10^{-4}$ \\
0.04 & $1.402 \times 10^{-3}$ \\
0.08 & $3.215 \times 10^{-3}$ \\
0.10 & $4.300 \times 10^{-3}$ \\
0.15 & $7.400 \times 10^{-3}$ \\
0.30 & $2.068 \times 10^{-2}$ \\
0.40 & $3.278 \times 10^{-2}$ \\
\hline
\end{tabular}


Table S-5. Influence of $n$-butylamine concentration for the aminolysis of NPA in chlorobenzene. $[15 \mathrm{C} 5]=0.10 \mathrm{M} ;[\mathrm{NPA}]=5 \times 10^{-5} \mathrm{M} . \mathrm{T}=25^{\circ} \mathrm{C}$.

\begin{tabular}{cc}
\hline $\left.\mathrm{BuNH}_{2}\right], \mathbf{M}$ & $\boldsymbol{k}_{\boldsymbol{o b s}}, \mathbf{s}^{-1}$ \\
\hline 0.02 & $1.751 \times 10^{-4}$ \\
0.04 & $3.916 \times 10^{-4}$ \\
0.08 & $1.069 \times 10^{-3}$ \\
0.10 & $1.449 \times 10^{-3}$ \\
0.15 & $2.824 \times 10^{-3}$ \\
0.30 & $9.448 \times 10^{-3}$ \\
0.40 & $1.691 \times 10^{-2}$ \\
\hline
\end{tabular}

Table S-6. Influence of $n$-butylamine concentration for the aminolysis of NPA in chlorobenzene. $[15 \mathrm{C} 5]=0.26 \mathrm{M} ;[\mathrm{NPA}]=5 \times 10^{-5} \mathrm{M} . \mathrm{T}=25^{\circ} \mathrm{C}$.

\begin{tabular}{cc}
\hline $\left.\mathrm{BuNH}_{2}\right], \mathbf{M}$ & $\boldsymbol{k}_{\text {obs }}, \mathbf{s}^{-1}$ \\
\hline 0.02 & $4.101 \times 10^{-4}$ \\
0.04 & $9.094 \times 10^{-4}$ \\
0.08 & $2.189 \times 10^{-3}$ \\
0.10 & $2.857 \times 10^{-3}$ \\
0.15 & $4.874 \times 10^{-3}$ \\
0.30 & $1.499 \times 10^{-2}$ \\
0.40 & $2.436 \times 10^{-2}$ \\
\hline
\end{tabular}


Table S-7. Influence of $n$-butylamine concentration for the aminolysis of NPA in chlorobenzene. $[15 \mathrm{C} 5]=0.51 \mathrm{M} ;[\mathrm{NPA}]=5 \times 10^{-5} \mathrm{M} . \mathrm{T}=25^{\circ} \mathrm{C}$.

\begin{tabular}{cc}
\hline $\left.\mathrm{BuNH}_{2}\right], \mathbf{M}$ & $\boldsymbol{k}_{\boldsymbol{o b s}}, \mathbf{s}^{-1}$ \\
\hline 0.02 & $7.997 \times 10^{-4}$ \\
0.04 & $1.824 \times 10^{-3}$ \\
0.08 & $4.115 \times 10^{-3}$ \\
0.10 & $5.257 \times 10^{-3}$ \\
0.15 & $9.082 \times 10^{-3}$ \\
0.30 & $2.414 \times 10^{-2}$ \\
0.40 & $3.799 \times 10^{-2}$ \\
\hline
\end{tabular}

Table S-8. Influence of $n$-butylamine concentration for the aminolysis of NPA in chlorobenzene. $[15 \mathrm{C} 5]=0.75 \mathrm{M} ;[\mathrm{NPA}]=5 \times 10^{-5} \mathrm{M} . \mathrm{T}=25^{\circ} \mathrm{C}$.

\begin{tabular}{cc}
\hline $\left.\mathrm{BuNH}_{2}\right], \mathbf{M}$ & $\boldsymbol{k}_{\text {obs }}, \mathbf{s}^{-1}$ \\
\hline 0.02 & $1.257 \times 10^{-3}$ \\
0.04 & $2.676 \times 10^{-3}$ \\
0.08 & $5.968 \times 10^{-3}$ \\
0.10 & $7.789 \times 10^{-3}$ \\
0.15 & $1.297 \times 10^{-2}$ \\
0.30 & $3.304 \times 10^{-2}$ \\
0.40 & $4.957 \times 10^{-2}$ \\
\hline
\end{tabular}


Table S-9. Influence of $n$-butylamine concentration for the aminolysis of NPA in chlorobenzene. $[18 \mathrm{C} 6]=0.10 \mathrm{M} ;[\mathrm{NPA}]=5 \times 10^{-5} \mathrm{M} . \mathrm{T}=25^{\circ} \mathrm{C}$.

\begin{tabular}{cc}
\hline $\left.\mathrm{BuNH}_{2}\right], \mathbf{M}$ & $\boldsymbol{k}_{\text {obs }}, \mathbf{s}^{-1}$ \\
0.02 & $3.173 \times 10^{-4}$ \\
0.08 & $1.780 \times 10^{-3}$ \\
0.10 & $2.171 \times 10^{-3}$ \\
0.15 & $3.845 \times 10^{-3}$ \\
0.18 & $5.991 \times 10^{-3}$ \\
0.22 & $7.390 \times 10^{-3}$ \\
0.30 & $1.144 \times 10^{-2}$ \\
\hline
\end{tabular}

Table S-10. Influence of $n$-butylamine concentration for the aminolysis of NPA in chlorobenzene. $[18 \mathrm{C} 6]=0.25 \mathrm{M} ;[\mathrm{NPA}]=5 \times 10^{-5} \mathrm{M} . \mathrm{T}=25^{\circ} \mathrm{C}$.

\begin{tabular}{cc}
\hline $\left.\mathrm{BuNH}_{2}\right], \mathbf{M}$ & $\boldsymbol{k}_{\text {obs }}, \mathbf{s}^{-1}$ \\
\hline 0.02 & $5.112 \times 10^{-4}$ \\
0.08 & $2.577 \times 10^{-3}$ \\
0.10 & $3.603 \times 10^{-3}$ \\
0.15 & $5.846 \times 10^{-3}$ \\
0.18 & $8.151 \times 10^{-3}$ \\
0.22 & $1.077 \times 10^{-2}$ \\
0.30 & $1.749 \times 10^{-2}$ \\
\hline
\end{tabular}


Table S-11. Influence of $n$-butylamine concentration for the aminolysis of NPA in chlorobenzene. $[18 \mathrm{C} 6]=0.50 \mathrm{M} ;[\mathrm{NPA}]=5 \times 10^{-5} \mathrm{M} . \mathrm{T}=25^{\circ} \mathrm{C}$.

\begin{tabular}{cc}
\hline $\left.\mathrm{BuNH}_{2}\right], \mathbf{M}$ & $\boldsymbol{k}_{\boldsymbol{o b s}}, \mathbf{s}^{-1}$ \\
\hline 0.02 & $1.047 \times 10^{-3}$ \\
0.08 & $4.868 \times 10^{-3}$ \\
0.10 & $6.374 \times 10^{-3}$ \\
0.15 & $1.062 \times 10^{-2}$ \\
0.18 & $1.351 \times 10^{-2}$ \\
0.22 & $1.777 \times 10^{-2}$ \\
0.30 & $2.763 \times 10^{-2}$ \\
\hline
\end{tabular}

Table S-12. Influence of $n$-butylamine concentration for the aminolysis of NPA in chlorobenzene. $[\mathrm{G} 2]=0.05 \mathrm{M} ;[\mathrm{NPA}]=5 \times 10^{-5} \mathrm{M} . \mathrm{T}=25^{\circ} \mathrm{C}$.

\begin{tabular}{cc}
\hline $\left.\mathrm{BuNH}_{2}\right], \mathbf{M}$ & $\boldsymbol{k}_{\text {obs }}, \mathbf{s}^{-1}$ \\
\hline 0.02 & $4.797 \times 10^{-5}$ \\
0.04 & $1.465 \times 10^{-4}$ \\
0.08 & $4.871 \times 10^{-4}$ \\
0.10 & $7.432 \times 10^{-4}$ \\
0.15 & $1.556 \times 10^{-3}$ \\
0.30 & $6.734 \times 10^{-3}$ \\
0.40 & $1.263 \times 10^{-2}$ \\
\hline
\end{tabular}


Table S-13. Influence of $n$-butylamine concentration for the aminolysis of NPA in chlorobenzene. $[\mathrm{G} 2]=0.11 \mathrm{M} ;[\mathrm{NPA}]=5 \times 10^{-5} \mathrm{M} . \mathrm{T}=25^{\circ} \mathrm{C}$.

\begin{tabular}{cc}
\hline $\left.\mathrm{BuNH}_{2}\right], \mathbf{M}$ & $\boldsymbol{k}_{\boldsymbol{o b s}}, \mathbf{s}^{-1}$ \\
\hline 0.02 & $6.225 \times 10^{-5}$ \\
0.04 & $1.746 \times 10^{-4}$ \\
0.08 & $5.620 \times 10^{-4}$ \\
0.10 & $8.424 \times 10^{-4}$ \\
0.15 & $1.706 \times 10^{-3}$ \\
0.30 & $7.214 \times 10^{-3}$ \\
0.40 & $1.354 \times 10^{-2}$ \\
\hline
\end{tabular}

Table S-14. Influence of $n$-butylamine concentration for the aminolysis of NPA in chlorobenzene. $[\mathrm{G} 2]=0.25 \mathrm{M} ;[\mathrm{NPA}]=5 \times 10^{-5} \mathrm{M} . \mathrm{T}=25^{\circ} \mathrm{C}$.

\begin{tabular}{cc}
\hline $\left.\mathrm{BuNH}_{2}\right], \mathbf{M}$ & $\boldsymbol{k}_{\text {obs }}, \mathbf{s}^{-1}$ \\
\hline 0.02 & $1.118 \times 10^{-4}$ \\
0.04 & $2.728 \times 10^{-4}$ \\
0.08 & $7.888 \times 10^{-4}$ \\
0.10 & $1.161 \times 10^{-3}$ \\
0.15 & $2.233 \times 10^{-3}$ \\
0.30 & $8.579 \times 10^{-3}$ \\
0.40 & $1.543 \times 10^{-2}$ \\
\hline
\end{tabular}


Table S-15. Influence of $n$-butylamine concentration for the aminolysis of NPA in chlorobenzene. $[\mathrm{G} 2]=0.48 \mathrm{M} ;[\mathrm{NPA}]=5 \times 10^{-5} \mathrm{M} . \mathrm{T}=25^{\circ} \mathrm{C}$.

\begin{tabular}{cc}
\hline $\left.\mathrm{BuNH}_{2}\right], \mathbf{M}$ & $\boldsymbol{k}_{\boldsymbol{o b s}}, \mathbf{s}^{-1}$ \\
\hline 0.02 & $1.918 \times 10^{-4}$ \\
0.04 & $4.415 \times 10^{-4}$ \\
0.08 & $1.147 \times 10^{-3}$ \\
0.10 & $1.607 \times 10^{-3}$ \\
0.15 & $3.007 \times 10^{-3}$ \\
0.30 & $1.037 \times 10^{-2}$ \\
0.40 & $1.839 \times 10^{-2}$ \\
\hline
\end{tabular}

Table S-16. Influence of $n$-butylamine concentration for the aminolysis of NPA in chlorobenzene. $[\mathrm{G} 2]=0.72 \mathrm{M} ;[\mathrm{NPA}]=5 \times 10^{-5} \mathrm{M} . \mathrm{T}=25^{\circ} \mathrm{C}$.

\begin{tabular}{cc}
\hline $\left.\mathrm{BuNH}_{2}\right], \mathbf{M}$ & $\boldsymbol{k}_{\text {obs }}, \mathbf{s}^{-1}$ \\
\hline 0.02 & $2.821 \times 10^{-4}$ \\
0.04 & $6.212 \times 10^{-4}$ \\
0.08 & $1.574 \times 10^{-3}$ \\
0.10 & $2.131 \times 10^{-3}$ \\
0.15 & $3.951 \times 10^{-3}$ \\
0.30 & $1.283 \times 10^{-2}$ \\
0.40 & $2.131 \times 10^{-2}$ \\
\hline
\end{tabular}


Table S-17. Influence of $n$-butylamine concentration for the aminolysis of NPA in chlorobenzene. $[\mathrm{G} 3]=0.05 \mathrm{M} ;[\mathrm{NPA}]=5 \times 10^{-5} \mathrm{M} . \mathrm{T}=25^{\circ} \mathrm{C}$.

\begin{tabular}{cc}
\hline $\left.\mathrm{BuNH}_{2}\right], \mathbf{M}$ & $\boldsymbol{k}_{\boldsymbol{o b s}}, \mathbf{s}^{-1}$ \\
\hline 0.02 & $1.997 \times 10^{-4}$ \\
0.04 & $4.666 \times 10^{-4}$ \\
0.08 & $1.124 \times 10^{-3}$ \\
0.10 & $1.554 \times 10^{-3}$ \\
0.15 & $2.750 \times 10^{-3}$ \\
0.30 & $9.230 \times 10^{-3}$ \\
0.40 & $1.506 \times 10^{-2}$ \\
\hline
\end{tabular}

Table S-18. Influence of $n$-butylamine concentration for the aminolysis of NPA in chlorobenzene. $[\mathrm{G} 3]=0.10 \mathrm{M} ;[\mathrm{NPA}]=5 \times 10^{-5} \mathrm{M} . \mathrm{T}=25^{\circ} \mathrm{C}$.

\begin{tabular}{cc}
\hline$\left[\mathrm{BuNH}_{2}\right], \mathbf{M}$ & $\boldsymbol{k}_{\text {obs }}, \mathbf{s}^{-1}$ \\
\hline 0.02 & $3.673 \times 10^{-4}$ \\
0.04 & $8.910 \times 10^{-4}$ \\
0.08 & $1.860 \times 10^{-3}$ \\
0.10 & $2.447 \times 10^{-3}$ \\
0.15 & $4.168 \times 10^{-3}$ \\
0.30 & $1.194 \times 10^{-2}$ \\
0.40 & $1.971 \times 10^{-2}$ \\
\hline
\end{tabular}


Table S-19. Influence of $n$-butylamine concentration for the aminolysis of NPA in chlorobenzene. $[\mathrm{G} 3]=0.25 \mathrm{M} ;[\mathrm{NPA}]=5 \times 10^{-5} \mathrm{M} . \mathrm{T}=25^{\circ} \mathrm{C}$.

\begin{tabular}{cc}
\hline$\left[\mathrm{BuNH}_{2}\right], \mathbf{M}$ & $\boldsymbol{k}_{\text {obs }}, \mathbf{s}^{-1}$ \\
0.02 & $9.321 \times 10^{-4}$ \\
0.04 & $1.933 \times 10^{-3}$ \\
0.08 & $4.161 \times 10^{-3}$ \\
0.10 & $5.328 \times 10^{-3}$ \\
0.15 & $8.663 \times 10^{-3}$ \\
0.30 & $2.110 \times 10^{-2}$ \\
0.40 & $3.176 \times 10^{-2}$ \\
\hline
\end{tabular}


Table S-20. Influence of $n$-butylamine concentration for the aminolysis of NPA in chlorobenzene. $[\mathrm{G} 4]=0.10 \mathrm{M} ;[\mathrm{NPA}]=5 \times 10^{-5} \mathrm{M} . \mathrm{T}=25^{\circ} \mathrm{C}$.

\begin{tabular}{cc}
\hline $\left.\mathrm{BuNH}_{2}\right], \mathbf{M}$ & $\boldsymbol{k}_{\text {obs }}, \mathbf{s}^{-1}$ \\
\hline 0.02 & $7.463 \times 10^{-4}$ \\
0.04 & $1.590 \times 10^{-3}$ \\
0.08 & $3.404 \times 10^{-3}$ \\
0.10 & $4.374 \times 10^{-3}$ \\
0.15 & $6.971 \times 10^{-3}$ \\
0.30 & $1.753 \times 10^{-2}$ \\
0.40 & $2.710 \times 10^{-2}$ \\
\hline
\end{tabular}

Table S-21. Influence of $n$-butylamine concentration for the aminolysis of NPA in chlorobenzene. [G4] $=0.25 \mathrm{M}$; $[\mathrm{NPA}]=5 \times 10^{-5} \mathrm{M} . \mathrm{T}=25^{\circ} \mathrm{C}$.

\begin{tabular}{cc}
\hline$\left[\mathrm{BuNH}_{2}\right], \mathbf{M}$ & $\boldsymbol{k}_{\boldsymbol{o b s}}, \mathbf{s}^{-1}$ \\
\hline 0.02 & $1.731 \times 10^{-3}$ \\
0.04 & $3.520 \times 10^{-3}$ \\
0.08 & $7.223 \times 10^{-3}$ \\
0.10 & $9.245 \times 10^{-3}$ \\
0.15 & $1.422 \times 10^{-2}$ \\
0.30 & $3.254 \times 10^{-2}$ \\
0.40 & $4.857 \times 10^{-2}$ \\
\hline
\end{tabular}


Table S-22. Influence of $n$-butylamine concentration for the aminolysis of NPA in chlorobenzene. $[\mathrm{G} 4]=0.50 \mathrm{M} ;[\mathrm{NPA}]=5 \times 10^{-5} \mathrm{M} . \mathrm{T}=25^{\circ} \mathrm{C}$.

\begin{tabular}{cc}
\hline $\left.\mathrm{BuNH}_{2}\right], \mathbf{M}$ & $\boldsymbol{k}_{\text {obs }}, \mathbf{s}^{-1}$ \\
\hline 0.02 & $3.325 \times 10^{-3}$ \\
0.04 & $6.797 \times 10^{-3}$ \\
0.08 & $1.398 \times 10^{-2}$ \\
0.10 & $1.785 \times 10^{-2}$ \\
0.15 & $2.769 \times 10^{-2}$ \\
0.30 & $5.995 \times 10^{-2}$ \\
0.40 & $8.413 \times 10^{-2}$ \\
\hline
\end{tabular}

Table S-23. Influence of $n$-butylamine concentration for the aminolysis of NPA in chlorobenzene. $[\mathrm{G} 4]=0.75 \mathrm{M} ;[\mathrm{NPA}]=5 \times 10^{-5} \mathrm{M} . \mathrm{T}=25^{\circ} \mathrm{C}$.

\begin{tabular}{cc}
\hline $\left.\mathrm{BuNH}_{2}\right], \mathbf{M}$ & $\boldsymbol{k}_{\text {obs }}, \mathbf{s}^{-1}$ \\
\hline 0.02 & $4.698 \times 10^{-3}$ \\
0.04 & $9.713 \times 10^{-3}$ \\
0.08 & $1.958 \times 10^{-2}$ \\
0.10 & $2.473 \times 10^{-2}$ \\
0.15 & $3.817 \times 10^{-2}$ \\
0.30 & $8.235 \times 10^{-2}$ \\
0.40 & $1.152 \times 10^{-1}$ \\
\hline
\end{tabular}


Table S-24. Influence of $n$-butylamine concentration for the aminolysis of NPA in chlorobenzene. $[\mathrm{G} 4]=1.00 \mathrm{M} ;[\mathrm{NPA}]=5 \times 10^{-5} \mathrm{M} . \mathrm{T}=25^{\circ} \mathrm{C}$.

\begin{tabular}{cc}
\hline$\left[\mathrm{BuNH}_{2}\right], \mathbf{M}$ & $\boldsymbol{k}_{\text {obs }}, \mathbf{s}^{-1}$ \\
0.02 & $6.018 \times 10^{-3}$ \\
0.04 & $1.216 \times 10^{-2}$ \\
0.08 & $2.480 \times 10^{-2}$ \\
0.10 & $3.229 \times 10^{-2}$ \\
0.15 & $4.636 \times 10^{-2}$ \\
0.30 & $1.029 \times 10^{-1}$ \\
0.40 & $1.444 \times 10^{-1}$ \\
\hline
\end{tabular}


Table S-25. Influence of $n$-butylamine concentration for the aminolysis of NPA in chlorobenzene. $[\mathrm{G} 5]=0.10 \mathrm{M} ;[\mathrm{NPA}]=5 \times 10^{-5} \mathrm{M} . \mathrm{T}=25^{\circ} \mathrm{C}$.

\begin{tabular}{cc}
\hline$\left[\mathrm{BuNH}_{2}\right], \mathbf{M}$ & $\boldsymbol{k}_{\text {obs }}, \mathbf{s}^{-1}$ \\
0.02 & $8.236 \times 10^{-4}$ \\
0.04 & $1.723 \times 10^{-3}$ \\
0.08 & $3.649 \times 10^{-3}$ \\
0.10 & $4.851 \times 10^{-3}$ \\
0.15 & $7.681 \times 10^{-3}$ \\
0.30 & $1.874 \times 10^{-2}$ \\
0.40 & $2.876 \times 10^{-2}$ \\
\hline
\end{tabular}

Table S-26. Influence of $n$-butylamine concentration for the aminolysis of NPA in chlorobenzene. $[\mathrm{G} 5]=0.26 \mathrm{M} ;[\mathrm{NPA}]=5 \times 10^{-5} \mathrm{M} . \mathrm{T}=25^{\circ} \mathrm{C}$.

\begin{tabular}{cc}
\hline $\left.\mathrm{BuNH}_{2}\right], \mathbf{M}$ & $\boldsymbol{k}_{\text {obs }}, \mathrm{s}^{-1}$ \\
\hline 0.02 & $1.996 \times 10^{-3}$ \\
0.04 & $4.108 \times 10^{-3}$ \\
0.08 & $8.524 \times 10^{-3}$ \\
0.10 & $1.089 \times 10^{-2}$ \\
0.15 & $1.680 \times 10^{-2}$ \\
0.30 & $3.797 \times 10^{-2}$ \\
0.40 & $5.520 \times 10^{-2}$ \\
\hline
\end{tabular}


Table S-27. Influence of $n$-butylamine concentration for the aminolysis of NPA in chlorobenzene. $[\mathrm{G} 5]=0.52 \mathrm{M} ;[\mathrm{NPA}]=5 \times 10^{-5} \mathrm{M} . \mathrm{T}=25^{\circ} \mathrm{C}$.

\begin{tabular}{cc}
\hline $\left.\mathrm{BuNH}_{2}\right], \mathbf{M}$ & $\boldsymbol{k}_{\boldsymbol{o b s}}, \mathbf{s}^{-1}$ \\
\hline 0.02 & $3.760 \times 10^{-3}$ \\
0.04 & $7.811 \times 10^{-3}$ \\
0.08 & $1.629 \times 10^{-2}$ \\
0.10 & $2.064 \times 10^{-2}$ \\
0.15 & $3.141 \times 10^{-2}$ \\
0.30 & $7.098 \times 10^{-2}$ \\
0.40 & $1.011 \times 10^{-1}$ \\
\hline
\end{tabular}

Table S-28. Influence of $n$-butylamine concentration for the aminolysis of NPA in chlorobenzene. $[\mathrm{G} 5]=0.75 \mathrm{M} ;[\mathrm{NPA}]=5 \times 10^{-5} \mathrm{M} . \mathrm{T}=25^{\circ} \mathrm{C}$.

\begin{tabular}{cc}
\hline$\left[\mathrm{BuNH}_{2}\right], \mathbf{M}$ & $\boldsymbol{k}_{\boldsymbol{o b s}}, \mathbf{s}^{-1}$ \\
\hline 0.02 & $5.799 \times 10^{-3}$ \\
0.04 & $1.173 \times 10^{-2}$ \\
0.08 & $2.384 \times 10^{-2}$ \\
0.10 & $3.048 \times 10^{-2}$ \\
0.15 & $4.650 \times 10^{-2}$ \\
0.30 & $1.003 \times 10^{-1}$ \\
0.40 & $1.401 \times 10^{-1}$ \\
\hline
\end{tabular}


Table S-29. Influence of $n$-butylamine concentration for the aminolysis of NPA in chlorobenzene. $[\mathrm{G} 5]=1.02 \mathrm{M} ;[\mathrm{NPA}]=5 \times 10^{-5} \mathrm{M} . \mathrm{T}=25^{\circ} \mathrm{C}$.

\begin{tabular}{cc}
\hline$\left[\mathrm{BuNH}_{2}\right], \mathbf{M}$ & $\boldsymbol{k}_{\text {obs }}, \mathbf{s}^{-1}$ \\
0.02 & $6.554 \times 10^{-3}$ \\
0.04 & $1.471 \times 10^{-2}$ \\
0.08 & $3.123 \times 10^{-2}$ \\
0.10 & $3.954 \times 10^{-2}$ \\
0.15 & $5.793 \times 10^{-2}$ \\
0.30 & $1.195 \times 10^{-1}$ \\
0.40 & $1.708 \times 10^{-1}$ \\
\hline
\end{tabular}


Table S-30. Influence of $n$-butylamine concentration for the aminolysis of NPA in chlorobenzene. $[\mathrm{G} 4]=0.10 \mathrm{M} ;[\mathrm{NPA}]=5 \times 10^{-5} \mathrm{M} . \mathrm{T}=-20^{\circ} \mathrm{C}$.

\begin{tabular}{cc}
\hline $\left.\mathrm{BuNH}_{2}\right], \mathbf{M}$ & $\boldsymbol{k}_{\boldsymbol{o b s}}, \mathbf{s}^{-1}$ \\
\hline 0.02 & $2.892 \times 10^{-4}$ \\
0.06 & $1.071 \times 10^{-3}$ \\
0.10 & $1.841 \times 10^{-3}$ \\
0.15 & $2.836 \times 10^{-3}$ \\
0.22 & $4.908 \times 10^{-3}$ \\
0.30 & $8.043 \times 10^{-3}$ \\
0.40 & $1.071 \times 10^{-2}$ \\
\hline
\end{tabular}

Table S-31. Influence of $n$-butylamine concentration for the aminolysis of NPA in chlorobenzene. [G4] $=0.25 \mathrm{M}$; $[\mathrm{NPA}]=5 \times 10^{-5} \mathrm{M} . \mathrm{T}=-20^{\circ} \mathrm{C}$.

\begin{tabular}{cc}
\hline $\left.\mathrm{BuNH}_{2}\right], \mathbf{M}$ & $\boldsymbol{k}_{\text {obs }}, \mathbf{s}^{-1}$ \\
\hline 0.02 & $7.660 \times 10^{-4}$ \\
0.06 & $2.459 \times 10^{-3}$ \\
0.10 & $4.804 \times 10^{-3}$ \\
0.15 & $7.122 \times 10^{-3}$ \\
0.22 & $1.081 \times 10^{-2}$ \\
0.30 & $1.667 \times 10^{-2}$ \\
0.40 & $2.083 \times 10^{-2}$ \\
\hline
\end{tabular}


Table S-32. Influence of $n$-butylamine concentration for the aminolysis of NPA in chlorobenzene. $[\mathrm{G} 4]=0.50 \mathrm{M} ;[\mathrm{NPA}]=5 \times 10^{-5} \mathrm{M} . \mathrm{T}=-20^{\circ} \mathrm{C}$.

\begin{tabular}{cc}
\hline $\left.\mathrm{BuNH}_{2}\right], \mathbf{M}$ & $\boldsymbol{k}_{\boldsymbol{o b s}}, \mathbf{s}^{-1}$ \\
\hline 0.02 & $1.498 \times 10^{-3}$ \\
0.06 & $4.456 \times 10^{-3}$ \\
0.10 & $9.025 \times 10^{-3}$ \\
0.15 & $1.329 \times 10^{-2}$ \\
0.22 & $2.015 \times 10^{-2}$ \\
0.30 & $2.796 \times 10^{-2}$ \\
0.40 & $3.995 \times 10^{-2}$ \\
\hline
\end{tabular}

Table S-33. Influence of $n$-butylamine concentration for the aminolysis of NPA in chlorobenzene. [G4] $=0.75 \mathrm{M}$; $[\mathrm{NPA}]=5 \times 10^{-5} \mathrm{M} . \mathrm{T}=-20^{\circ} \mathrm{C}$.

\begin{tabular}{cc}
\hline $\left.\mathrm{BuNH}_{2}\right], \mathbf{M}$ & $\boldsymbol{k}_{\boldsymbol{o b s}}, \mathbf{s}^{-1}$ \\
\hline 0.02 & $2.084 \times 10^{-3}$ \\
0.06 & $7.330 \times 10^{-3}$ \\
0.10 & $1.295 \times 10^{-2}$ \\
0.15 & $1.866 \times 10^{-2}$ \\
0.22 & $2.831 \times 10^{-2}$ \\
0.30 & $3.921 \times 10^{-2}$ \\
0.40 & $5.644 \times 10^{-2}$ \\
\hline
\end{tabular}


Table S-34. Influence of $n$-butylamine concentration for the aminolysis of NPA in chlorobenzene. $[\mathrm{G} 4]=1.00 \mathrm{M} ;[\mathrm{NPA}]=5 \times 10^{-5} \mathrm{M} . \mathrm{T}=-20^{\circ} \mathrm{C}$.

\begin{tabular}{cc}
\hline $\left.\mathrm{BuNH}_{2}\right], \mathbf{M}$ & $\boldsymbol{k}_{\text {obs }}, \mathbf{s}^{-1}$ \\
0.02 & $2.766 \times 10^{-3}$ \\
0.06 & $8.884 \times 10^{-3}$ \\
0.10 & $1.438 \times 10^{-2}$ \\
0.15 & $2.353 \times 10^{-2}$ \\
0.22 & $3.347 \times 10^{-2}$ \\
0.30 & $4.730 \times 10^{-2}$ \\
0.40 & $6.583 \times 10^{-2}$ \\
\hline
\end{tabular}


Table S-35. Influence of $n$-butylamine concentration for the aminolysis of NPA in chlorobenzene. $[\mathrm{G} 4]=0.10 \mathrm{M} ;[\mathrm{NPA}]=5 \times 10^{-5} \mathrm{M} . \mathrm{T}=45^{\circ} \mathrm{C}$.

\begin{tabular}{cc}
\hline $\left.\mathrm{BuNH}_{2}\right], \mathbf{M}$ & $\boldsymbol{k}_{\boldsymbol{o b s}}, \mathbf{s}^{-1}$ \\
\hline 0.02 & $7.462 \times 10^{-4}$ \\
0.06 & $2.522 \times 10^{-3}$ \\
0.10 & $4.806 \times 10^{-3}$ \\
0.15 & $8.169 \times 10^{-3}$ \\
0.22 & $1.401 \times 10^{-2}$ \\
0.30 & $2.346 \times 10^{-2}$ \\
0.40 & $3.919 \times 10^{-2}$ \\
\hline
\end{tabular}

Table S-36. Influence of $n$-butylamine concentration for the aminolysis of NPA in chlorobenzene. $[\mathrm{G} 4]=0.25 \mathrm{M} ;[\mathrm{NPA}]=5 \times 10^{-5} \mathrm{M} . \mathrm{T}=45^{\circ} \mathrm{C}$.

\begin{tabular}{cc}
\hline $\left.\mathrm{BuNH}_{2}\right], \mathbf{M}$ & $\boldsymbol{k}_{\text {obs }}, \mathbf{s}^{-1}$ \\
\hline 0.02 & $1.857 \times 10^{-3}$ \\
0.06 & $6.075 \times 10^{-3}$ \\
0.10 & $1.089 \times 10^{-2}$ \\
0.15 & $1.900 \times 10^{-2}$ \\
0.22 & $3.026 \times 10^{-2}$ \\
0.30 & $4.614 \times 10^{-2}$ \\
0.40 & $6.578 \times 10^{-2}$ \\
\hline
\end{tabular}


Table S-37. Influence of $n$-butylamine concentration for the aminolysis of NPA in chlorobenzene. $[\mathrm{G} 4]=0.50 \mathrm{M} ;[\mathrm{NPA}]=5 \times 10^{-5} \mathrm{M} . \mathrm{T}=45^{\circ} \mathrm{C}$.

\begin{tabular}{cc}
\hline $\left.\mathrm{BuNH}_{2}\right], \mathbf{M}$ & $\boldsymbol{k}_{\text {obs }}, \mathbf{s}^{-1}$ \\
\hline 0.02 & $3.702 \times 10^{-3}$ \\
0.06 & $1.171 \times 10^{-2}$ \\
0.10 & $2.178 \times 10^{-2}$ \\
0.15 & $3.531 \times 10^{-2}$ \\
0.22 & $5.234 \times 10^{-2}$ \\
0.30 & $8.058 \times 10^{-2}$ \\
0.40 & $1.072 \times 10^{-1}$ \\
\hline
\end{tabular}

Table S-38. Influence of $n$-butylamine concentration for the aminolysis of NPA in chlorobenzene. $[\mathrm{G} 4]=0.75 \mathrm{M} ;[\mathrm{NPA}]=5 \times 10^{-5} \mathrm{M} . \mathrm{T}=45^{\circ} \mathrm{C}$.

\begin{tabular}{cc}
\hline $\left.\mathrm{BuNH}_{2}\right], \mathbf{M}$ & $\boldsymbol{k}_{\text {obs }}, \mathbf{s}^{-1}$ \\
\hline 0.02 & $5.423 \times 10^{-3}$ \\
0.06 & $1.781 \times 10^{-2}$ \\
0.10 & $3.223 \times 10^{-2}$ \\
0.15 & $4.935 \times 10^{-2}$ \\
0.22 & $7.724 \times 10^{-2}$ \\
0.30 & $1.062 \times 10^{-1}$ \\
0.40 & $1.579 \times 10^{-1}$ \\
\hline
\end{tabular}


Table S-39. Influence of $n$-butylamine concentration for the aminolysis of NPA in chlorobenzene. $[\mathrm{G} 4]=1.00 \mathrm{M} ;[\mathrm{NPA}]=5 \times 10^{-5} \mathrm{M} . \mathrm{T}=45^{\circ} \mathrm{C}$.

\begin{tabular}{cc}
\hline$\left[\mathrm{BuNH}_{2}\right], \mathbf{M}$ & $\boldsymbol{k}_{\text {obs }}, \mathbf{s}^{-1}$ \\
0.02 & $7.258 \times 10^{-3}$ \\
0.06 & $2.411 \times 10^{-2}$ \\
0.10 & $4.004 \times 10^{-2}$ \\
0.15 & $6.249 \times 10^{-2}$ \\
0.22 & $9.626 \times 10^{-2}$ \\
0.30 & $1.387 \times 10^{-1}$ \\
0.40 & $1.966 \times 10^{-1}$ \\
\hline
\end{tabular}


Table S-40. Influence of $n$-butylamine concentration for the aminolysis of NPA in chlorobenzene. $[\mathrm{NPA}]=5 \times 10^{-5} \mathrm{M} . \mathrm{T}=25^{\circ} \mathrm{C}$.

\begin{tabular}{cc}
\hline $\left.\mathrm{BuNH}_{2}\right], \mathbf{M}$ & $\boldsymbol{k}_{\text {obs }}, \mathbf{s}^{-1}$ \\
0.02 & $1.780 \times 10^{-3}$ \\
0.06 & $3.836 \times 10^{-3}$ \\
0.10 & $6.339 \times 10^{-3}$ \\
0.15 & $9.265 \times 10^{-3}$ \\
0.22 & $1.427 \times 10^{-2}$ \\
0.30 & $2.058 \times 10^{-2}$ \\
0.40 & $2.806 \times 10^{-2}$ \\
\hline
\end{tabular}

